Research Article

\title{
Effect of Type 2 Diabetes Mellitus on Cognitive function and EEG in elderly patients
}

\author{
Dr.S.Bethiun ${ }^{1}$, Dr.R.Premaraja ${ }^{2}$ \\ ${ }^{1}$ Assistant professor of Physiology, Sri Balaji Medical College and Hospital, Chennai \\ ${ }^{2}$ Assistant professor of Physiology, Sri Lakshmi Narayana Institute of Medical Sciences, Puducherry
}

\begin{abstract}
:
Introduction: Diabetes is a major health problem in India, Type 2 is also the most common type seen. Diabetes is associated with cognitive decline in elderly patients. Hyperglycemia, age, sex and duration of diabetes mellitus all seems to affect cognitive function.

Objectives: Aim of the study is to assess the EEG changes and cognitive function in elderly type 2 diabetes mellitus patients.

Materials and methods: Two groups of people, 20 in each group who are age sex matched were selected and case group of elderly diabetic patients and control group of healthy subjects were selected. EEG recordings were done using MP36 BIOPAC 4 channel instrument and cognitive function was assessed using MMSE.

Results: The elderly diabetic group was having declined cognitive function - MCI (Mild Cognitive Impairment) and reduced EEG wave activity.

Conclusion: Reduced EEG alpha wave activity is seen in elderly diabetic patients. Reduced functional connections in cortex and hyperglycemia induced pathologic changes could be the causative factors.
\end{abstract}

Keywords: Diabetes Mellitus, EEG, Cognition, Elderly

\section{INTRODUCTION}

Type 2 diabetes constitutes more than $97 \%$ of the diabetes mellitus patients. 1 Prevalence of diabetes mellitus is from ${ }^{1}$ to $50 \%$ in South Asian population among different ethnicities. ${ }^{2,3}$ Diabetes in South India is also rapidly increasing in incidence. ${ }^{4}$ Number of people with diabetes is predicted to rise from 171 million to 366 million in 2030., 5 Type 2 diabetes is related with cognitive dysfunction in elderly patients. There is increased risk of dementia and cognitive impairment including Alzheimer's disease. ${ }^{5-7}$

Cognitive dysfunction in diabetes mellitus is explained using causative role by hyperglycemia, insulin resistance, hypoglycemia and amyloid resistance. ${ }^{8}$ Cognitive impairment such as learning and memory deficiency was seen in type 2 diabetes. ${ }^{9}$ The diabetic individuals have increased risk of Mild cognitive impairment. ${ }^{8-11}$ It is also affected by patient's age, type of diabetes, type of therapy and comorbidities. Hyperglycemia affects brain function by modification of polyol pathway, formation of advanced glycation end products, activation of protein kinase $\mathrm{C}$ and increased hexosamine shunt pathway.

Mini-Mental state examination done in patients had found correlation between age, sex, duration of diabetes mellitus with cognitive function. EEG changes were seen in type 2 diabetes mellitus patients with hyperglycemic episodes. $5,6,9,10$

There are very few studies documenting EEG changes compared to cognitive tests involving mental tests.

\section{Objectives}

Aim of the study was to assess the cognitive function and EEG changes in elderly patients with Type 2 diabetes mellitus.

1) Assess the cognitive function in both case and control groups

2) Record and analyse the EEG in Case and Control groups

3) Compare the cognitive functions and EEG in between the case and control groups

\section{Methodology}

This study was designed as a hospital based case-control study. Patients attending the outpatient department and patients admitted in the Sri Lakshmi Narayana Institute of Medical Sciences, who were Type 2 Diabetes Mellitus patients were randomly selected and Mini-Mental state examination was done to assess cognitive function among the patients. In the same patients Electroencephalogram was done to assess EEG changes. Patients were matched by age and gender. EEG recording was done using BIOPAC 4 channel instrument in Department of Physiology.

Slow wave activity in EEG was compared between the cases and controls. Slow wave activity was shown to be increased in patients with type 2 diabetes mellitus. ${ }^{8}$ Theta and delta activity in EEG was increased in the patients with type 2 diabetes mellitus. ${ }^{8}$ These were compared in the EEG recording done in cases and controls. 


\section{Dr. R. Premaraja et al / Effect of Type 2 Diabetes Mellitus on Cognitive function and EEG in elderly patients}

Data was collected from both elderly patients with type 2 diabetes mellitus and normal subjects in the same age group. The cognitive function and EEG changes were compared with both the subjects and control group.

Study design - Case - Control study

Sample size: 20 cases and 20 controls

Inclusion Criteria: Patients above the age of 50 years and having type 2 diabetes for 5 years and on treatment.

Controls: healthy subjects in the same age group

Exclusion criteria: Any other co-morbid conditions, depression, learning impairment, hypertension, stroke, epilepsy, coronary vascular diseases and renal diseases.

Subjects were instructed to wash their hair on the day of investigation and to avoid oil, hair creams and conditioners and to avoid caffeinated beverages and smoking on the day of test.

Data was collected using the mini mental state examination (MMSE) and EEG recording done using the BIOPAC 4 channel system (MP36, Made in USA).

Mini Mental State Examination (MMSE) is 30 point scoring system which measures orientation, registration - immediate memory, short term memory and language functioning. Patients were made to sit comfortably and after establishing rapport the mini mental state examination was done. Scores were calculated for each subject and compared between case and control groups.

EEG recording was done in frontal \& parieto-occipital region for theta rhythm and in frontal \& temporo-parietal region for delta rhythm. ${ }^{8}$ Bipolar leads were used for the recording. Measurements were done with amplitudes expressed in microvolts and frequency in hertz. Measurements were taken after the subjects are seated and relaxed in a quiet room. SS2L electrode lead set and gel surface electrodes were used on the scalp. Appropriate instructions are given to the subjects prior to the recording. Recording were done with subject awake and both eyes closed and opened states. These measurements were used for EEG analysis.

Informed consent was taken from the patients and controls for the study.

Statistical analysis: Data recorded were grouped into cases and controls and analysed with Independent sample " $t$ " test as a test of significance and a $\mathrm{P}$ value less 0.05 will be used as significant.

\section{Results}

The two groups were matched in, age, sex and education level.(Table 1) There were significant differences in scores of MMSE between cases and controls.(Table 2) (1) In comparison with controls, patients with Mild cognitive impairment (MCI) had a marked decrease in lower alpha, upper alpha, and beta bands. Lower functional connections at short and long intra-hemispheric distance may have contributed to this around left hemisphere. (2) In the lower alpha band was significantly lower in MCI group.

Table 1: Mean age, diabetes duration, cognitive status and the number for the participants of each group enrolled in the present study

\begin{tabular}{|l|l|l|l|l|l|}
\hline Group & $\begin{array}{l}\text { Number } \\
\text { and Sex }\end{array}$ & $\begin{array}{l}\text { Age in } \\
\text { years }\end{array}$ & $\begin{array}{l}\text { Education } \\
\text { in years }\end{array}$ & $\begin{array}{l}\text { Diabetes } \\
\text { duration } \\
\text { in years }\end{array}$ & MMSE \\
\hline Case & $\begin{array}{l}20 \\
(10 \mathrm{M}+10 \mathrm{~F})\end{array}$ & $62.6 \pm 4.3$ & $10.5 \pm 2.1$ & $5.9 \pm 3.4$ & $26.8 \pm 0.6$ \\
\hline Control & $\begin{array}{l}20 \\
(10 \mathrm{M}+10 \mathrm{~F})\end{array}$ & $63.5 \pm 5.2$ & $14.4 \pm 2.5$ & -- & $28.6 \pm 0.4$ \\
\hline
\end{tabular}

Table 2: Neuropsychological test scores and $\mathbf{P}$ values between Diabetes and control groups

\begin{tabular}{|l|l|l|l|}
\hline Factor & Case group & Control group & P value \\
\hline MMSE scores & $26.8 \pm 0.6$ & $28.6 \pm 0.4$ & $0.034 *$ \\
\hline
\end{tabular}

\section{Discussion}

Older individuals with type 2 diabetes have slow EEG activity in central cortex and reduced alpha wave activity in parietal area, compared with age-matched nondiabetics they have decreased relative alpha power, suggesting effects of hyperglycemia. High blood glucose level in type 2 diabetes could be the main causative factor for EEG abnormalities.

\section{Conclusions}

The brain network of MCI patients displayed a disconnection syndrome and a loss of interconnections. The correlation between cognitive states and network characteristics suggested that the more in deterioration of the diabetes patients' cognitive state, the less optimal the network organization become. Hence, the complex network-derived biomarkers based on EEG could be employed to track cognitive function of diabetic patients and provide a new diagnosis tool for aMCI.

\section{References}

[1] AdeghateE, Schattner P, Dunn E. An update on the etiology and epidemiology of diabetes mellitus. Ann N Y Acad Sci. 2006 Nov 28;1084:1-29.

[2] Vaz NC, Ferreira A, Kulkarni M, Vaz FS, Pinto N. Prevalence of Diabetic Complications in Rural Goa, Indian J Community Med. 2011 Oct-Dec; 36(4): 283 286.

[3] Zimmet P. Type II (non insulin dependent diabetes) - an epidemiological overview. Diabetologia. 1982; 22: 399411.

[4] Monika G, Prabhu K, Parijatham O. Prevalence of Diabetes Mellitus in South India: A Retro spective Analysis, JIMSA October-December 2012 Vol. 25 No. $4: 238-240$ 
[5] Miles WR, Root HF 1922 Psychologic tests applied to diabetic patients. Arch Intern Med 30:767-777.

[6] Baskaran A, Milev R. A review of electroencephalographic changes in diabetes mellitus in relation to major depressive disorder. Neuropsychiatric disease and treatment. 2013;9: 143-150.

[7] Eeg-Olofsson O, Petersen I. Childhood diabetic neuropathy: a clinical and neurophysiological study. Acta Paediatr Scand Suppl. 1966;55: 163-176.

[8] Kumar V., Nelson F., Abbas A. K., Cotran R. S., Robbins S. L. (2005). Robbins and Cotran Pathologic Basis of Disease, 7th Edn. Philadelphia, PA: Saunders

[9] Peila R., Rodriguez B. L., Launer L. J. (2002). Type 2 diabetes, APOE gene, and the risk for dementia and related pathologies: the Honolulu-Asia Aging Study. Diabetes 51, 1256-1262

[10] Shimada H., Miki T., Tamura A., Ataka S., Emoto M., Nishizawa Y. (2010). Neuropsychological status of elderly patients with diabetes mellitus. Diabetes Res. Clin. Pract. 87, 224-227

[11] Roberts R. O., Kantarci K., Geda Y. E., Knopman D. S., Przybelski S. A., Weigand S. D., et al. (2011). Untreated type 2 diabetes and its complications are associated with subcortical infarctions. Diabetes Care 34, 184-186

[12] Jelic V., Shigeta M., Julin P., Almkvist O., Winblad B., Wahlund L. O. (1996). Quantitative electroencephalography power and coherence in Alzheimer's disease and mild cognitive impairment. Dementia 7, 314-323 